\title{
Parental occupational exposure to benzene and the risk of childhood and adolescent acute lymphoblastic leukaemia: a population-based study
}

\author{
Julia E Heck, 1,2,3 Di He, ${ }^{1}$ Zuelma Arellano Contreras, ${ }^{1}$ Beate Ritz, ${ }^{1,2}$ Jørn Olsen, 1,4 \\ Johnni Hansen ${ }^{5}$
}

- Additional material is published online only. To view please visit the journal online (http://dx.doi.org/10.1136/ oemed-2019-105738).

'Department of Epidemiology, Fielding School of Public Health, University of California, Los Angeles, Los Angeles, California, USA

${ }^{2}$ Center for Occupational and Environmental Health, University of California, Los Angeles, CA, USA

${ }^{3}$ Jonsson Comprehensive Cancer Center, University of California, Los Angeles, CA, USA

${ }^{4}$ Department of Clinical Epidemiology, Aarhus University, Aarhus, Denmark

Institute of Cancer

Epidemiology, Danish Cance

Society, Copenhagen, Denmark

Correspondence to

Dr Julia E Heck, Epidemiology, University of California, Los Angeles, CA 90095, USA; jeheck@ucla.edu

Received 30 January 2019

Revised 26 April 2019

Accepted 5 May 2019

Published Online First

28 May 2019

\begin{abstract}
Objectives Only a small number of studies have reported on the association of parental occupational exposure to benzene and risk of childhood and adolescent leukaemias. We examined associations with acute lymphoblastic leukaemia (ALL) in this populationbased study in Denmark.
\end{abstract}

Methods Benzene was largely banned from Danish workplaces after 1975, thus this case-control study focused on the immediately prior years. Paediatric cancer cases (<age 20) were ascertained from the Danish Cancer Registry among children born 1968-1974, and controls were selected from population records. Paternal occupation within the 3 months preconception and maternal pregnancy occupation were identified from nationwide pension fund records. Blinded, we assigned benzene exposure using a job-exposure matrix that had been developed for the Danish population. Risk for ALL was estimated using conditional logistic regression. In an exploratory analysis, we also examined other cancers with at least five case parents exposed.

Results We identified 217 employed case fathers and 169 employed case mothers, of which 22 $(10.1 \%)$ and $11(6.5 \%)$, respectively, were exposed to benzene (vs $6.7 \%$ and $2.9 \%$ of control fathers and mothers). Most exposed parents worked as machine or engine mechanics, or in the shoe industry. Maternal occupational exposure to benzene in pregnancy was related to increased risk of ALL in offspring (adjusted $\mathrm{OR}=2.28,95 \% \mathrm{Cl} 1.17$ to 4.41 ), while paternal preconceptional benzene exposure was not as strongly associated (adjusted OR=1.40, 95\% Cl 0.88 to 2.22).

Conclusions Our study supports an increased risk for ALL with parental occupational benzene exposure.

\section{INTRODUCTION}

Benzene has been encountered in a variety of occupational settings as an organic solvent and in engine exhaust originating from $1 \%$ to $5 \%$ levels in gasoline. The International Agency for Research on Cancer classified benzene as carcinogenic to humans (Group 1) based on increased risk of acute myeloid leukaemia in adults. ${ }^{1}$ Childhood and adolescent acute lymphoblastic leukaemia (ALL) has been studied less frequently, and results were mixed (OR range: mothers 0.5-2.6; fathers $0.8-2.0) .^{2-5}$ One study was population-based, using record-linkage of national registries, ${ }^{5}$ while the other studies collected data from parental interviews. Most of these studies applied job-exposure

\section{Key messages}

What is already known about this subject?

- Benzene has been encountered in a variety of occupational settings as an organic solvent and in engine exhaust originating from 1 to $5 \%$ levels in gasoline.

- The International Agency for Research on Cancer has classified benzene as carcinogenic to humans (Group 1) based upon increased risk of acute myeloid leukaemia in occupational studies of adults. Less is known about leukaemia risk in offspring from parental exposure to benzene.

What are the new findings?

- Maternal occupational exposure to benzene in pregnancy was related to increased risk of acute lymphoblastic leukaemia (ALL) in offspring.

- Compared to maternal exposure, paternal preconceptional benzene exposure was less strongly associated with increased risk of ALL.

\section{How might this impact on policy or clinical} practice in the foreseeable future?

- This study's focus on benzene, independent of other chemicals, provides support for lower benzene in engine exhausts and other workplace exposures.

matrices (JEMs) to assess exposure, while one relied on parental recall of chemicals encountered at work. As such, some previous findings may have been subject to recall bias or selective participation. Further, few studies had adequate specificity of exposure assessment to identify a potential effect from benzene independent of exposure to other chemicals; this is relevant given that persons exposed to benzene also may have high exposure to toluene and other agents. We sought to examine ALL risk from parental occupational exposure to benzene in a population-based study in which exposure was objectively estimated by expert assessment.

\section{METHODS}

As previously described, ${ }^{6}$ we identified incident cases of ALL less than 20 years of age from the Danish Cancer Registry, then randomly selected controls among those matched by sex and age (25:1 matching rate) from the 
source population. Parental occupations during the perinatal period were identified from the Supplementary Pension Fund, ${ }^{7}$ a compulsory supplement to the state pension for all employed persons age 18-66. The Supplementary Pension Fund records the start and end dates of all paid jobs along with the industry code, a five-digit extended version of the International Standard Industrial Classification of All Economic Activities. All information is maintained by the Supplementary Pension Fund even when businesses close or in the case of a person's death. Occupations with benzene exposure (ever/never exposed) were identified using the Danish version of the Nordic Occupational Cancer Study JEM. ${ }^{8}$ Because workplace benzene use began to drop prior to the first study year and our JEM assigned few workplace benzene exposures after 1975, we limited analyses to children born 1968-1974.

We excluded from the present analysis parents without records of employment (stay-at-home parents, students and self-employed persons, whose records are not in the Pension Fund) during the perinatal period ( $23 \%$ of case fathers and $40 \%$ of case mothers). However, we conducted a sensitivity analysis to evaluate the effect of excluding these parents, in which we considered these parents as unexposed. Blinded to case-control status, we estimated risk of ALL from parental occupational exposure to benzene, examining fathers' exposures in the 3 months preconception and maternal exposures in pregnancy. Gestational age information was taken from the Medical Births Registry as previously described. ${ }^{6}$ We did not estimate cancer risk from both parents being occupationally exposed to benzene, because this was extremely rare.

We estimated ORs and 95\% CIs using conditional logistic regression with adjustment for parental ages and socioeconomic status. Inclusion of parental age in models was based on the literature as well as associations seen in our data. ${ }^{6}$ We additionally considered adjustment for socioeconomic status because it was related to the exposure. We were not able to adjust for maternal smoking because it was not collected during the time period of interest; however, a recent study of Danish children, based on the same national registries, did not report any increased risk of ALL from smoking. ${ }^{9}$ Further, adjustment for socioeconomic status will also partly adjust for smoking. We additionally examined associations after the exclusion of children with Down syndrome.

Because benzene was highly correlated with toluene ${ }^{1}$ we could not easily determine whether any observed associations with benzene might also have been due to toluene. As such we separately examined the risk of ALL from occupational toluene exposure during the time period immediately after benzene use was largely phased out (1975-1984).

In an exploratory analysis we also examined other cancer types with at least five exposed cases. There were six (8.6\%) exposed astrocytoma case fathers, $10(10.9 \%)$ exposed fathers of germ cell tumour cases and five (22.7\%) exposed case fathers of a rare leukaemia subtype, acute undifferentiated leukaemia/acute leukaemia not otherwise specified (NOS; International Classification of Diseases for Oncology (ICD-O-1) code 98 013). All analyses were done using SAS 9.4.

\section{RESULTS}

We observed excesses of ALL for maternal exposure to benzene in pregnancy (table 1). With regards to paternal preconception exposure, ORs were elevated with wide CIs. The parents in our study exposed to benzene were most often employed as machine and engine mechanics, with smaller numbers employed in laboratory settings, in the shoe industry, or in other manufacturing (online Supplementary table 1). The exclusion of children with Down syndrome did not change results appreciably (online Supplementary table 2). When we considered parents without employment records as unexposed to benzene and included them in analyses, results did not change (online Supplementary table 3).

In examining the association of exposure to toluene between 1975 and 1984 and risk of ALL, we did not observe associations between ALL and paternal preconception toluene exposure (online Supplementary table 4; adjusted OR $=1.14,95 \%$ CI 0.78 to 1.68) nor maternal pregnancy toluene exposure (adjusted $\mathrm{OR}=0.57,95 \% \mathrm{CI} 0.23$ to 1.41 ).

Paternal benzene exposure was not clearly related to astrocytoma (online Supplementary table 5; adjusted OR $=1.29,95 \% \mathrm{CI}$ 0.54 to 3.06), germ cell tumours (adjusted $\mathrm{OR}=1.37,95 \% \mathrm{CI} 0.69$ to 2.71), but was related to acute undifferentiated leukaemia/acute leukaemia NOS (adjusted OR=6.11, 95\% CI 1.81 to 20.61).

\section{DISCUSSION}

In this population-based study in which parental occupational benzene exposure was assessed via JEM, we observed increases in ALL with maternal exposure to benzene in pregnancy. In Denmark and internationally, the proportion of benzene in gasoline has dropped considerably over the last decades, with current European Union regulations limiting benzene to less than $1 \%$ of gasoline. ${ }^{1}$ In Denmark, its use as a solvent in other occupational settings was phased out by the mid-1970s. Workplace exposures are expected to have been considerably more widespread in the 1950s and 1960s, prior to the study period. ${ }^{1}$

Our results are in line with most other studies on maternal ${ }^{235}$ and paternal $^{3-5}$ occupational benzene and ALL which showed increased effect estimates for the pregnancy period and preconception. Of the studies that examined maternal exposures, most relied on parental interview $^{2-4}$ while one utilised parental occupation as listed on census records and linked to cancer registry data. ${ }^{5}$ Point estimates were elevated across most studies, although CIs were often wide due to small numbers of exposed parents. Not all studies showed increases in risk, but results may have been influenced by the use of proxy respondents (usually the mother reporting on father's exposures), moderate participation rates, or unequal socioeconomic distributions between case and control groups.

Table 1 Parental occupational exposure to benzene and risk of childhood and adolescent acute lymphoblastic leukaemia among children born 1968-1974

\begin{tabular}{|c|c|c|c|c|c|}
\hline & N Exposed/total cases & N Exposed/total controls & Crude OR & Adjusted $\mathrm{OR}^{*}(95 \% \mathrm{Cl})$ & Adjusted ORt $(95 \% \mathrm{Cl})$ \\
\hline \multicolumn{6}{|c|}{ Father's occupations in 3 months preconception } \\
\hline Any occupation with benzene exposure & $22 / 217(10.1 \%)$ & $400 / 5233(6.7 \%)$ & $1.42(0.89,2.24)$ & $1.42(0.90$ to 2.25$)$ & $1.40(0.88$ to 2.22$)$ \\
\hline \multicolumn{6}{|l|}{ Mother's occupations during pregnancy } \\
\hline Any occupation with benzene exposure & $11 / 169(6.5 \%)$ & $112 / 3905(2.9 \%)$ & $2.28(1.18,4.39)$ & 2.31 (1.19 to 4.63$)$ & $2.28(1.17$ to 4.41$)$ \\
\hline
\end{tabular}

${ }^{*}$ Adjusted for maternal and paternal age.

†Adjusted for maternal and paternal age and family socioeconomic status. 
The preconception and pregnancy periods are plausibly relevant time periods to examine the influence of parental exposures. ALL is likely initiated in utero, as shown by studies of neonatal dried blood spots which have identified ALL-related leucocyte chromosomal translocations in children who later developed leukaemia. ${ }^{10}$ Benzene metabolites induce genomic instability and a variety of chromosomal aberrations, ${ }^{1}$ thus it is feasible that they could cause the characteristic translocations seen in ALL.

We did not attempt to assess narrower time periods of exposure (eg, trimesters) since few parents changed jobs during pregnancy. In the 1970 s, maternity leave in Denmark officially began 8 weeks prior to the expected due date, although in practice it could have started earlier if the mother's health concerns necessitated it. Thus, the maternal benzene exposures that we estimated would have occurred primarily in the first and second trimesters. Although benzene metabolites can be transferred in breast milk, exposure level would have likely dropped after birth during maternity leave and because in the late 1960s and early 1970s, half of all Danish mothers stayed home with their small children. ${ }^{11}$ Families would have had other sources of benzene exposure due to traffic exhaust and because European Union directives restricting benzene concentration in toys did not occur until 1982 (Directive 82/806/EEC).

Advantages of this study include the record-based, population-based design with the inclusion of all cancer cases born and diagnosed in Denmark during the time period of interest, thus without selection bias. We used a validated JEM to assess exposures. Our reliance on employment records with the start and end dates of each position meant our study was not subject to recall bias. A limitation of our study is that the use of a JEM will inherently introduce non-differential misclassification of exposure, which may bias results towards the null. The small numbers of exposed parents preluded us examining dose-response in results.

In sum, we observed increases in ALL risk among children whose parents were occupationally exposed to benzene. Given evidence of a linear association between ambient benzene exposure and childhood and adolescent leukaemia, ${ }^{12}$ it is justified to reduce occupational benzene exposures.
Twitter @uclafsph @uclajccc@cancer_dk @ucla_coeh

Contributors JEH conceived, designed the presented work and drafted the article. $\mathrm{JH}$ acquired and linked data. DH and ZAC performed the data analysis. All authors reviewed results, commented on the manuscript and approved of the final version to be published.

Funding This study was supported by grants from the US National Institutes of Health (R21CA175959, R03ES021643). JEH was supported from a grant from Alex's Lemonade Stand Foundation (grant 17-01882).

Competing interests None declared.

Patient consent for publication Not required.

Ethics approval Human subjects approvals for this study were received from the Danish Data Protection Board and the University of California, Los Angeles, California, USA.

Provenance and peer review Not commissioned; externally peer reviewed.

\section{REFERENCES}

1 IARC Monographs Program. Benzene. WHO Press: Lyon, 2018.

2 Infante-Rivard C, Siemiatycki J, Lakhani R, et al. Maternal exposure to occupational solvents and childhood leukemia. Environ Health Perspect 2005;113:787-92.

3 Shu XO, Stewart P, Wen WQ, et al. Parental occupational exposure to hydrocarbons and risk of acute lymphocytic leukemia in offspring. Cancer Epidemiol Biomarkers Prev 1999:8:783-91.

4 Metayer C, Scelo G, Kang AY, et al. A task-based assessment of parental occupational exposure to organic solvents and other compounds and the risk of childhood leukemia in California. Environ Res 2016;151:174-83.

5 Spycher BD, Lupatsch JE, Huss A, et al. Parental occupational exposure to benzene and the risk of childhood cancer: A census-based cohort study. Environ Int 2017; 108:84-91.

6 Contreras ZA, Hansen J, Ritz B, et al. Parental age and childhood cancer risk: a Danish population-based registry study. Cancer Epidemiol 2017;49:202-15.

7 Hansen J, Lassen CF. The Supplementary Pension Fund Register. Scand J Public Health 2011:39:99-102.

8 Kauppinen T, Heikkilä P, Plato N, et al. Construction of job-exposure matrices for the Nordic Occupational Cancer Study (NOCCA). Acta Oncol 2009;48:791-800.

9 Momen NC, Olsen J, Gissler M, et al. Exposure to maternal smoking during pregnancy and risk of childhood cancer: a study using the Danish national registers. Cancer Causes Control 2016;27:341-9.

10 Greaves MF, Wiemels J. Origins of chromosome translocations in childhood leukaemia Nat Rev Cancer 2003;3:639-49.

11 Rostgaard T, Christoffersen MN, Weise H. Parental leave in Denmark. In: Moss P, Deven F, eds. Parental leave: progress or pitfall? Research and policy issues in Europe. Brussels: NIDI/CBGS Publications, 1999.

12 Filippini T, Hatch EE, Rothman KJ, et al. Association between outdoor air pollution and childhood leukemia: a systematic review and dose-response meta-analysis. Environ Health Perspect 2019;127:046002. 